\title{
Über Ermüdung willkürlich oder elektrisch gereizter Muskeln.
}

Von

Fritz Uhlmann, prakt. Arzt, ans Trub.

(Mit 14 Textfiguren.)

Die Ermüdung und Erholung isolierter Froschmuskeln hat H. K r o necker ${ }^{1}$ ) gründlich untersucht. Er fand schon vor 40 Jabren :

1. Die Ermüdungskurve (Verbindungslinie der Hubgipfel) des in gleichen Zeitintervallen mit gleich starken (maximalen) Induktionsschlägen gereizten „überlasteten“ (Helmholtz) Muskels ist eine gerade Linie.

2. Die Differenz der Ermüdungsreihe (Steilheit des Abfalles) nimmt $a b$, wenn die Reizintervalle wachsen.

3. Die Steilheit des Ermüdungsabfalles ändert sich nicht mit den Belastungen. Kleine Gewichte werden zwar höher gehoben als grosse, aber die verschiedenen Ermüdungslinien verlaufen einander parallel.

In Übereinstimmung mit anderen Pbysiologen zeigte $\mathrm{K}$ r o n e ck er, dass Blutzirkulation die Ermüdung verzögert.

Angelo $\mathrm{M} \mathrm{os} \mathrm{s}^{2}$ ) hat vor 20 Jahren mittels seines Ergographen am Menschen die Ermüdung der Musc. flexor. digit. sublimis et profundus aufzeichnen lassen und damit die Anregung zu zahlreichen physiologischen und psychologischen Versuchsreihen gegeben.

Er konnte bei ergographischen Versuchen mit Aduceo und Maggiora durch elektrische Reizung des N. medianus nicht so starke Kontraktion des Mittelfingerbeugers erzielen wie durch willkürliche Aktion. Kronecker fand bei seinen Ermüdungsversuchen (O. C. S. 261), dass, wenn die Wadenmuskeln eines unversehrten

1) Arbeiten aus der physiol. Anstalt zu Leipzig. Jahrg. 1871. Leipzig 1872.

2) Arch. ital. de Biol. t. 13 p. 124. Turin 1890. Les Lois de la fatigue étudiées dans les muscles de l'homme. 
Frosches durch 11/4 stündige Reizfolge (720 Zuckungen) fast vollständig erschöpft waren, das freigelassene Tier sich nicht willkürlich zu bewegen vermochte. Nach 21 Stunden war es noch sehr lahm. Die Gastrocnemii machten hiernach, auch wenn sie maximal elektrisiert wurden, nur noch 320 niedere Zuckungen. Das Tier war noch nach 2 Tagen, gleich einem kuraresierten, zusammengi'sunken, unfähig zu willkürlicher Bewegung.

Die ergographischen Versuche von Mosso und seinen Schülern (a. a. O.) ergaben, wie schon oben erwähnt, dass der Mittelfinger willkürlich noch ein grosses Gewicht wiederholt zu heben vermochte, nachdem elektrische Reizung des $\mathrm{N}$. medianus unwirksam geworden war.

Mosso schloss daraus, dass auch die Ermüdung durch intensive Gehirnarbeit wesentlich die peripheren Muskeln betreffe (S. 156). R. Mülle $\mathbf{r}^{x}$ meint, dass die willkürliche Anregung die Musculi i uterossei in Tätigkeit versetze, während künstliche Reizung des N. medianus die Flexores digit. communes ermüde.

Grützne ${ }^{2}$ ) nimmt an, dass bei den mannigfaltigen natürlichen Muskeltätigkeiten stets einzelne Fasern verschiedener Muskelindividuen innerviert und zur Zusammenziehung gebracbt werden. Er vermochte auch durch künstliche Reizmittel verschiedene flinke oder träge Fasern in demselben Muskel getrennt zu erregen. Schwache Indüktionsströme erzeugen im Froschsartorius durch Reizung der dünnen, trägen Fasern sehr niedrigen, glatten Tetanus; starke Induktionsströme verursachen in den dicken, flinken Fasern intermittierende Kontraktionen (Zittern). Willkürliche Bewegungen sollen mannigfache Frregungen verschiedener Fasergruppen auslösen.

Als $M o s s 0^{3}$ ) einen N. medianus von Herrn $A d u c c o$ rbythmisch elektrisierte, ermüdeten die Fingerflexoren nicht für willkürliche Reize. Aber "während der Muskel willkürlich arbeitete, rehabilitierte er sich nicht wieder für den Nervenreiz".

Die willkürlichen wie auch die auf elektrischen Reiz erfolgenden Zusammenziehungen der Muskeln des lebenden Menschen waren niemals maximal.

1) Über Mosso's Ergographen mit Rücksicht auf seine physiologischen und psychologischen Anwendungen. Wundt's Philosoph. Studien 1901.

2) Bericht über den VI. internationalen Physiologenkongress zu Brüssel. Arch. intern. de Physiol. de Frederic et Heger 1904-1905 p. 87.

3) Über die Gesetze der Ermüdung. Du Bo is-Reymond's Arch. 1890 S. 128. 
Tiege ${ }^{1}$ ) fand bei untermaximal gereizten Froschmuskeln folgenden Unterschied in der Ermüdung, gegenüber der maximal gereizten: „Während der Muskel bei irgendeinem untermaximalen Reize arbeitet, erholt er sich für jeden grösseren Reiz, mag derselbe nun maximal oder unmaximal sein. Wenn der Muskel aber maximal zuckt, so kann nie, in keinem Stadium seiner Ermủdung, durch Reizverstärkung eine Erhöhung der Zuckungen erreicht werden" (Kronecker).

Maggi o $\mathrm{ra}^{2}$ ) batte gefunden, dass man durch geeignete Kombination der Arbeitsgrössen (3 kg) und Erholungspausen (10 Sek.) einen Muskel unermüdet behalten kann.

Broca und Richet ${ }^{3}$ ) fanden für den willkürlich kontrahierten Flexor indicis maximale Arbeitswerte bei Hebung von etwa $1600 \mathrm{~g}$ in Intervallen von 1 bis 2 Sekunden.

L. Treves ${ }^{4}$ ) vermochte mit gebeugtem Mittelfinger das Maximum zu leisten: wenn er $6 \mathrm{~kg} 28 \mathrm{mal}$ in 1 Minute hob. Er erreichte damit den Arbeitswert von $243 \mathrm{~kg} / \mathrm{m}$ in 1 Stunde. Er kommt zum Schlusse: „Pour un même poids, l'énergie qu'on peut atteindre dans le travail rythmique est d'autant plus grande que le rythme est plus lent." Um die teilweise widersprechenden Angaben zu kontrollieren, mussten die Versuche mit exakten Methoden wiederholt werden ${ }^{5}$ ). Mir diente hierzu folgendes Verfahren:

1) Über den Einfluss einiger willkürlich Veränderlichen auf die Zuckungshöhe des untermaximal gereizten Muskels. Arbeiten aus der physiol. Anstalt zu Leipzig Bd. 10 S. 19. 1875. Ber. der math.-phys. Klasse der kgl. sächs. Gesellsch, der Wissensch. zu Leipzig Bd. 27 S. 99.

2) Les lois de la fatigue étudiées dans les muscles de l'homme. Arch. ital. de Biol. t. 13 p. 199.1890.

3) Ergographie. Dictionnaire de Physiol. t, 6 p. 5381.

4) L'énergie de contraction dans le travail musculaire volontaire et la fatigue nerveuse. Arch. de fisiol. da Fano t. 1 p. 190/192.

5) Die früher üblichen Ergographen, nach Angelo Mosso's Modell, verzeichnen die Bewegungen des gebeugten Mittel- oder Zeigefingers (DuboisSchnyder), bei festgestellter Hand. Von der gebeugten Anfangsstellung aus wurde der weitere Flexionsakt registriert. Die Fingerbeuger arbeiteten also mehr oder weniger belastet. Im gespannten Zustande vermochten sie grössere Gewichte $\mathrm{zu}$ tragen $(3-8 \mathrm{~kg})$. Unser horizontal gelagerter Vorderarm spannte den Bizeps immer in gleicher sehr geringer Weise. Daher arbeitet er wesentlich überlastet, und so vermag daher die mächtige Muskelgruppe nur geringe Gewichte $(1-4 \mathrm{~kg}) \mathrm{zu}$ heben. 
Die Ermüdung meines M. biceps brachii registrierte ich vermittels eines Ermüdungsschreibers, den wir "Ka mat og ra ph" nennen. Derselbe ist nach den Angaben von Professor Kronecker in den Werkstätten des Herrn Bandagisten S chenk konstruiert und in der Telegraphenwerkstätte $\mathrm{H}$ a s l er A.-G. B er n, vervollkommnet worden.

Herr Cutter ${ }^{1}$ ) (aus New York) hat im Jahre 1900 die Ermüdung sowie die Trainierung am gleichen Apparate in dessen primitiver Form untersucht. Er fand, dass mässiges Gehen oder leichtes Steigen (300 m) 25-40 Minuten o h ne S to ck (also ohne Gebrauch der Armmuskeln) seinen Biceps brachii weder ermüdete noch stärkte. Ruhiges Bergsteigen während 1 bis 2 Stunden stärkte seine Arme ein wenig. Ermüdende Besteigungen um $300 \mathrm{~m}$ und mehr während 10 bis 14 Stunden minderten für 2 bis 3 Tage die Hubfähigkeit seines Armbeugers. Am vierten Tage nach der Anstrengung war dagegen die Kraft seiner Arme derart vermebrt, dass die erste Versuchsreihe bis 80 Hebungen ergab.

\section{Beschreibung des Kamatographen.}

Die Versuchsperson sitzt, wie Fig. 3 illustriert, auf einem Drehsessel neben dem Apparate oder liegt auf einem Operationstische. Im ersten Falle ist die dem Apparate zugewendete Achselhöhle durch eine gepolsterte Krücke $A$ gestützt, die, entsprechend der Länge des Oberarms, eingestellt wird. Vgl. 1 und 2.

Der horizontal gerichtete Vorderarm ruht auf einer gepolsterten Rinne $B$. Ein passend einzustellender Holzblock $C$ fixiert den Oberarm.

Wenn die Versuchsperson horizontal gelagert ist, so ruben Oberarm und Vorderarm auf der Rinne. Um das Handgelenk ist eine Ledermanschette $D$ geschnallt. Von dieser geht ein Strick $E$ zu einem schienenförmigen Doppelhebel $F$, der um die Achse $G$ drehbar ist. Auf dieser eisernen Schiene ist ein Gewicht $H$ verschiebbar, das den Arm mit $500 \mathrm{~g}$ bis $\mathrm{zu} 4 \mathrm{~kg}$ belasten kann. Damit der Angriffstpunkt in konstanter Entfernung vom Handgelenke gehalten werde, läuf die Schnur in einer bogenförmigen Rinne $I$. Der Radius dieser Rinue ist gleich der Entfernung des Hebelendes von seinem Drehpunkte.

1) Effets du travail de certains groupes musculaires sur d'autres groupes qui ne font aucun travail. Compt. rend. de Académie des sciences. Paris, 3. Sept. 1900. 
Über Ermüdung willkürlich oder elektrisch gereizter Muskeln. $\quad 521$

Wenn der Gewichtshebel $F$ gedreht wird, so rotiert auf gleicher Achse ein Zahnrad $K$. Die Zähne dieses Randes greifen in diejenigen eines zweiten $K_{2}$ von gleichem Durchmesser $(12 \mathrm{~cm})$. Um die Hebung des Gewichtes auf das Papier $P$ zu notieren, ist mit der Acbse des Rades $K_{2}$ (Fig. 2) der Winkelhebel $L_{1} L_{2}$ verbunden,

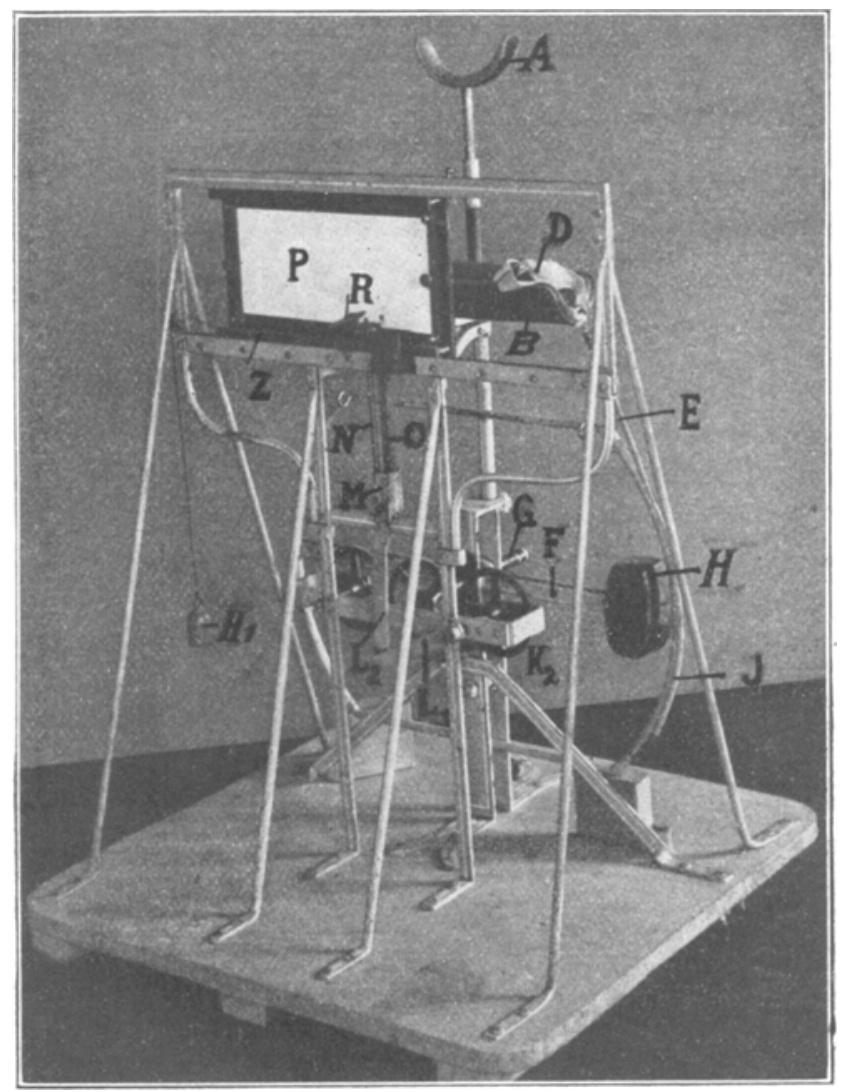

Fig. 1. (Von vorne gesehen.)

dessen vertikal geführter Schenkel $L_{2}$ den Bleistifthalter an der vierkantigen Leitstange $O$ hinaufführt. Der Schieber $M$ sichert den vertikalen Gang. Der Bleistift $R$ (ferdernd an die $35 \mathrm{~cm}$ lange und $15 \mathrm{~cm}$ hohe Schreibfläche $P$ gedrückt) verzeichnet die Hubhöhen der Hand. Das Verhältnis der wirklichen Hubhöhen des Gewichtes zu denjenigen, welche auf die Schreibfäche gezeichnet werden, ändert sich mit der Entfernung des Gewichts vom Drehpunkte. Für die 
von uns verwendeten Gewichte von $1,2,3$ und $4 \mathrm{~kg}$ verhilelten sich die wirklichen Hubhöhen $\mathrm{zu}$ den verzeichneten wie folgt: für $1 \mathrm{~kg}=1 / 2$, für $2 \mathrm{~kg}=1 / 1$, für $3 \mathrm{~kg}=3 / 2$, für $4 \mathrm{~kg}=2 / 1$.

Bei den folgenden Tabellen ist diesen Maassverhältnissen Rechnung getragen. Es sind die auf der Schreibfläche verzeichneten

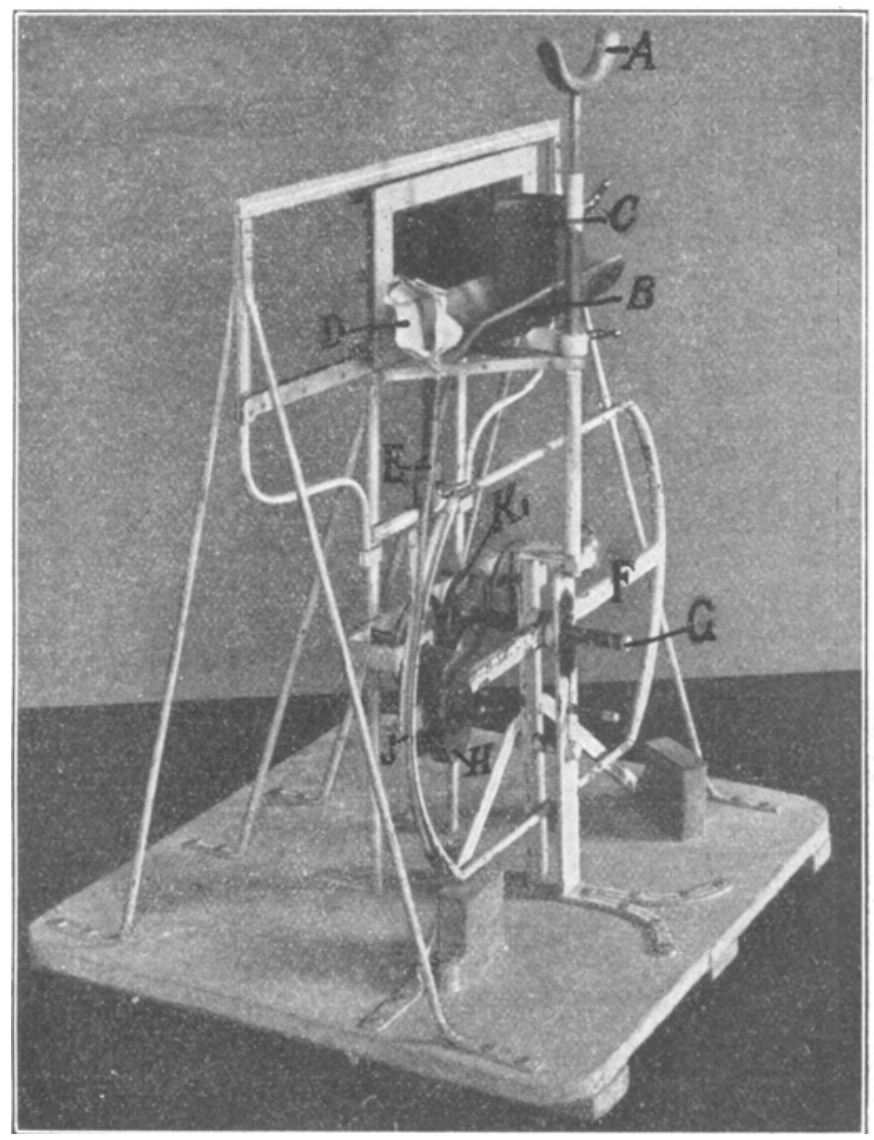

Fig. 2. (Von hinten und seitlich gesehen.)

Hubhöhen in die wirklichen umgerechnet. Wenn der Bleistifthalter $N$ zurückfällt, so greift der auf seinem Rande befestigte Sperrzahn in ein Sperrad im Gehäuse $e$ (Fig. 1) ein. Dieser ist konaxial mit einem Triebe verbunden, der auf einer langen Zahnstange $Z$ rollt. Ein kleines Zuggewicht $H$, das den Schreibflächerahmen rückwärts hält, verhindert, dass das Sperrad bei jedem Hube mehr als einen Zahn der Stange springen lässt. 
Um auch die Arbeit des linken Armes aufzeichnen zu können, ist ein drittes Zahnrad angebracht, das gleich dem zweiten vom Mittelrade getrieben wird. Es wird für diesen Fall das Schiebgewicht auf die andere Hälfte des Doppelhebels gesetzt und das untere Ende des Winkelhebels mit der Achse des letzten Rades verbunden. Auf diesem Ende des Hebels kann die zweite Kreisrinne den Strick aufnehmen, welcher dann von der linken Handwurzel gehoben wird.

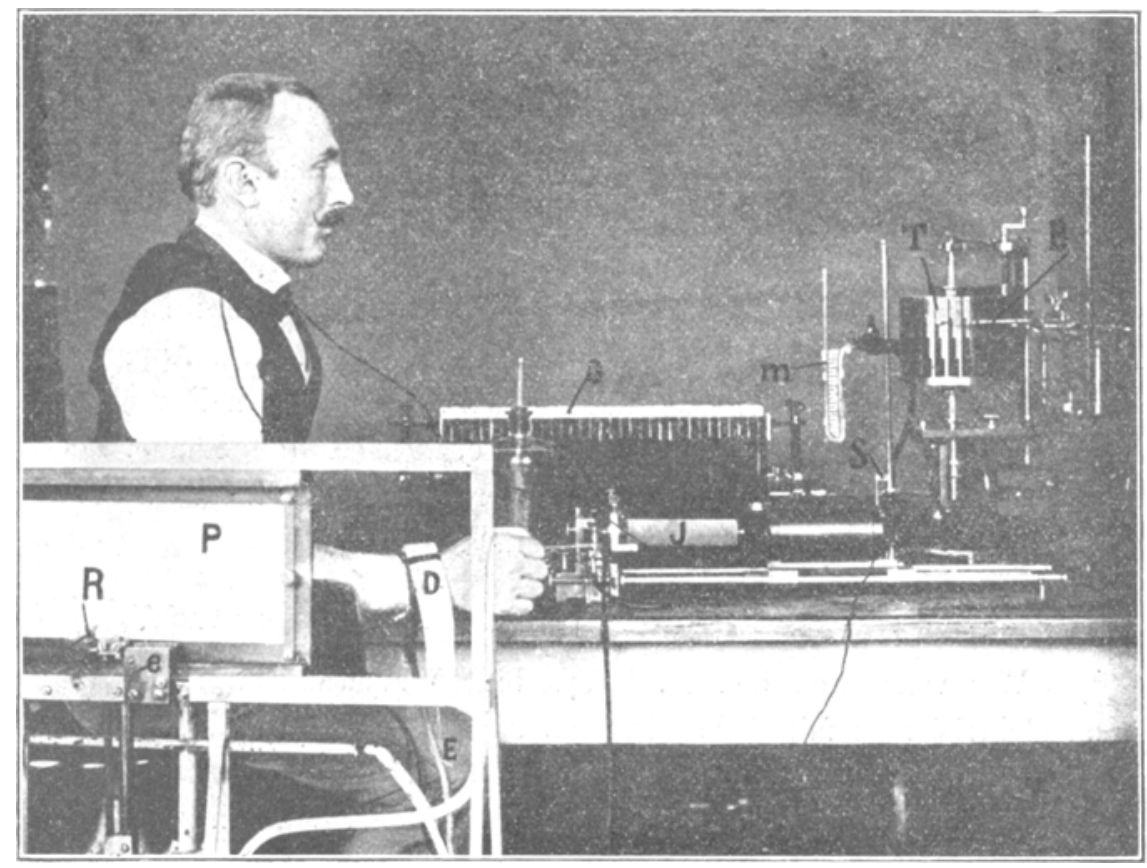

Fig. 3.

Methodik.

Zunächst mussten wir die Versuchsbedingungen sichern: in erster Linie die Intensitäten der Muskelreize. Wenn wir die Willensreize durch genau messbare elektrische ersetzen wollten, waren die in Fig. 3 photographierten Apparate erforderlich.

\section{Arten elektrischer Reizung.}

Schliessung wie Öffnung galvanischer Ströme bewirkte, auch bei schmerzhafter Intensität, keine Muskelkontraktion von merklicher Höhe, Auch lange Funken (bis $20 \mathrm{~cm}$ ) einer Influenzmaschine waren 
motorisch unwirksam, während sie schmerzten und Brandwunden gaben. Intermittierenide Induktionsströme hatten Erfolg. Um genau bestimmbare Reize zu erhalten, verwendeten wir ein grosses Schlitteninduktorium ${ }^{1}$ ), dessen primäre Spirale den sehr konstanten Strom einer Gülcher'chen Thermosäule $(G)$ von 66 Elementen aufnahm. Dieselbe liefert, bei mittlerem Gasdrucke, eine Spannung von 3 Volt und bewahrt dieselbe, was wesentlich ist, bei gleichmässiger Erwärmung tagelang. Den Gasdruck kontrollierten wir mittels eines neben die Gasleitung eingeschalteten Wassermanometers $(m)$. Der Gasdruck wurde auf $30 \mathrm{~cm}$ Wasser gehalten. Das Schlitteninduktorium war nach Stromeinheiten graduiert.

Die Unterbrechungen des Primärstromes konnten innerhalb der Frequenzen 25 bis 100 in der Sekunde variiert werden. Ein Spülkontakt hielt die Widerstände im primären Stromkreise konstant. Wenn wir seltene Interruptionen zu erhalten wünschten, so verwendeten wir Kronecker's Unterbrecher. In diesem Apparate schwingt ein eisernes Lineal, durch Elektromagneten in Bewegung erhalten. Fin verschiebbarer Halter ermöglicht die Länge des schwingenden Linealabschnittes zu ändern. Die Schwingungszahlen waren von 4 bis 60 in 1 Sekunde veränderlich.

\section{Die Applikation der Ströme auf den Biceps brachii.}

Wir versuchten den M. biceps brachii zunïchst direkt bipolar zu elektrisieren: mittels bepolsterter, angefeuchteter Metallknopfpole, welche durch $z$ wei Leinenstreifen auf den Muskelbauch geschnallt wurden. Diese Anordnung genügte nicht; daher stachen wir zwei versilberte Nähnadeln tief in meinen zweiköpfigen Armmuskel ein. Auch diese schmerzhafte Zuleitungsart hatte nicht den gewünschten Erfolg. Wir bemerkten (gleich Mosso), dass sich nur diejenigen Muskelbündel kontrahierten, welche in der Verbindungslinie der beiden Elektroden oder nahe daran lagen. Die erzielte Leistung des Muskels war unter diesen Umständen sehr gering. Wir versuchten dann mittels unipolarer Reizung $\mathrm{zu}$ wirken. Die mit feuchter Leinwand umwickelte kupferne Knopfelektrode war uber die Mitte des Bizepbauches gebunden, während die indifferente, plattenförmige hepolsterte Elektrode auf der Bauchhaut in der Nabelgegend lag. Auch bei dieser Anordnung blieb schmerzhafte

1) Beschreibung siehe Zeitschrift für Instrumentenkunde 1902. 
Reizung ohne genügende Muskelwirkung. Wir gelangten so schliesslich zu der Überzeugung, dass es nicht möglich sei, durch direkte Reizung den gesamten Muskel zur Kontraktion zu bringen. Daher wandten wir uns zur Nervenreizung. Wenn die Elektrode in der Medianusrinne lag, litt ich während der Reizung heftige Schmerzen, sobald die Ströme zu erwünschter motorischer Kraft gesteigert wurden. Wir suchten endlich die zwei sogenannten "motorischen Punkte" (D uchenne) ${ }^{1}$ ) über dem Bizeps, entsprechend der oberen Teilung des N. musculocutaneus, und erhielten mit intermittierenden Induktionsströmen sehr kräftige Tetani. Günstig erwies sich der etwas oberhalb der Mitte des Muskelbauches gelegene Punkt. Der höhere und mehr nach der Achsel zu gelegene war nicht gut zu gebrauchen, weil von hier aus häufig der Stamm des N. musculocutaneus mit seinen sensiblen Fasern gereizt wurde. Der brauchbare Punkt musste .also gut eingehalten werden: Nachdem ich den best-motorischen und mindest-sensiblen Reizpunkt hatte, suchten wir die günstigste Form und Grösse der wirksamen Elektrode. Am besten bewährte sich als Reizelektrode ein kreisförmiges vernickeltes Kupferblech von $15 \mathrm{~mm}$ Durchmesser und 0,5 mm Dicke. Eine in der Mitte angelötete Klemmschraube nahm den Leitungsdraht auf. Ein darunter gelegtes nasses Wattebäuschchen hielt die Reizstelle feucht. Zur Anfeuchtung benutzten wir schwach angesäuertes Wasser. Fixiert wurde dieses Plättchen durch gekreuzte Heftpflasterstreifen. Die indifferente Elektrode: ein rechteckiges Zinkblech von 9:12 cm Seiten lag mit angefeuchtetem Polster, durch den Beinkleidergurt fixiert, auf der Nabelgegend. Die Reizelektrode, mittels Pipette jede Viertelstunde angefeuchtet, blieb während langer Versuchsreihen oft tagelang auf den Arm geklebt. So sparte ich die Zeit zur Aufsuchung des Reizpunktes. Auch die indifferente Elektrode musste von Zeit zu Zeit in Versuchspausen frisch angefeuchtet werden. Mit dem Bizeps kontrahierte sich stets auch der M. brachialis, weil beide Muskeln von dem gereizten N. musculocutaneus versorgt werden.

III. Dauer und Rhythmus der tetanisierenden Reize.

Wir machten die Reizkombinationen, bei welcher der Muskel am meisten arbeitet.

1) Sahli, Lehrbuch der klinischen Untersuchungsmethoden, 4. Aufl., S. 833 . 1905 . 
1. "Reizdauer": die Zeit, welche verstreicht vom Beginne der tetanisierenden Reizung bis zur maximalen Kontraktion.

2. Als "Intervall" bezeichnen wir die Zeit zwischen den Tetani.

3. Unter "Ru h epa use" verstehen wir die Erholungsfristen des Muskels zwischen den Reizgruppen.

Zur rhythmischen Reizfolge balf uns folgendes Verfahren:

Die Metalitrommel eines $L \mathbf{u} d \mathbf{w}$ i $g$ 'schen Kymographions liessen wir mit vertikalen Streifen isolierenden Asphaltlackes bemalen. In Fig. 4 sieht man die abgestuften Streifen angedeutet, wenn man sich den Zylindermantel des Kymographions (vgl. $T$ Fig. 3) abgerollt vorstellt. Die Trommel hat einen Umfang von $50 \mathrm{~cm}$; und es befanden

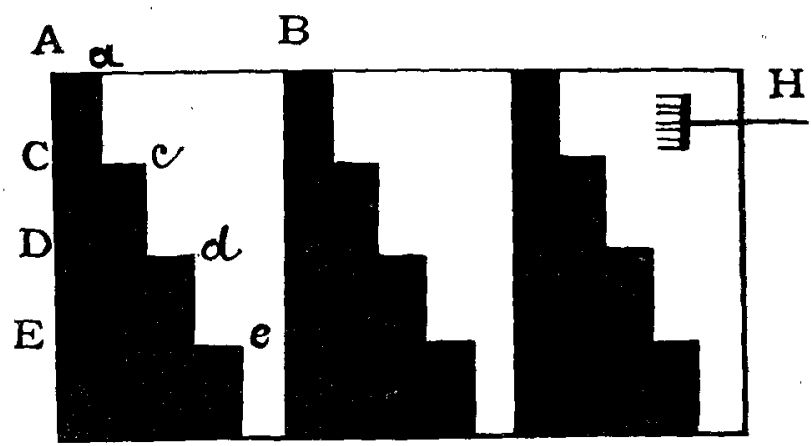

Fig. 4. (Breitedimensionen natürl. Grösse.)

sich 25 solcher Streifen darauf, so dass die Strecke $A B 2 \mathrm{~cm}$ betrug. $A a=\frac{A B}{5}=4 \mathrm{~mm} ; C c=8 \mathrm{~mm} ; D d=12 \mathrm{~mm} ; E e=16 \mathrm{~mm}$.

Der eine Pol der den Biceps reizenden sekundären Spirale des Induktoriums war mit den Metallteilen des Kymographions verbunden, während der andere $\mathrm{Pol}$ mit der Metallbürste $H$ über die rotierende Trommel strich. Solange die Bürste über freie Metallabschnitte des Zylinders ging, waren die tetanisierenden Induktionsströme durcb metalliscbe Nebenschliessung vom Arme abgeblendet, während die Bürste aber über die isolierenden Streifen glitt, war der Strom in der zweiten Leitung, welche durch den Körper ging, frei. Die Abblendung des Stromes war so vollständig, dass ich, während die Bürste auf den Metallteilen des Zylinders Nebenschluss machte, nicht das geringste fühlte. Der besseren Übersicht wegen seien hier noch kurz die Verbindungen schematisiert. Vgl. Fig. 5. 
Wir prüften zunächst die Reizdauer von 0,5 Sek. und regulierten daher die Geschwindigkeit der Trommel derart, dass die Bürste 0,5 Sek. über die Strecke $A a$ (Fig. 4) strich. Da die Strecke $A a$ $1 / 5$ von $A B$ und $A B$ wieder $1 / 25$ vom ganzen Umfang der Trommel, so musste letztere einen Umgang in 1 Min. und 2,5 Sek. vollenden. Auf diese Weise erhielten wir folgende Kombination: 0,5 Sek. Reiz, 2 Sek. Intervall. Wurde nun die Trommel höher gestellt, während die Bürste stehenblieb, so dass letztere die Zonen II oder III oder

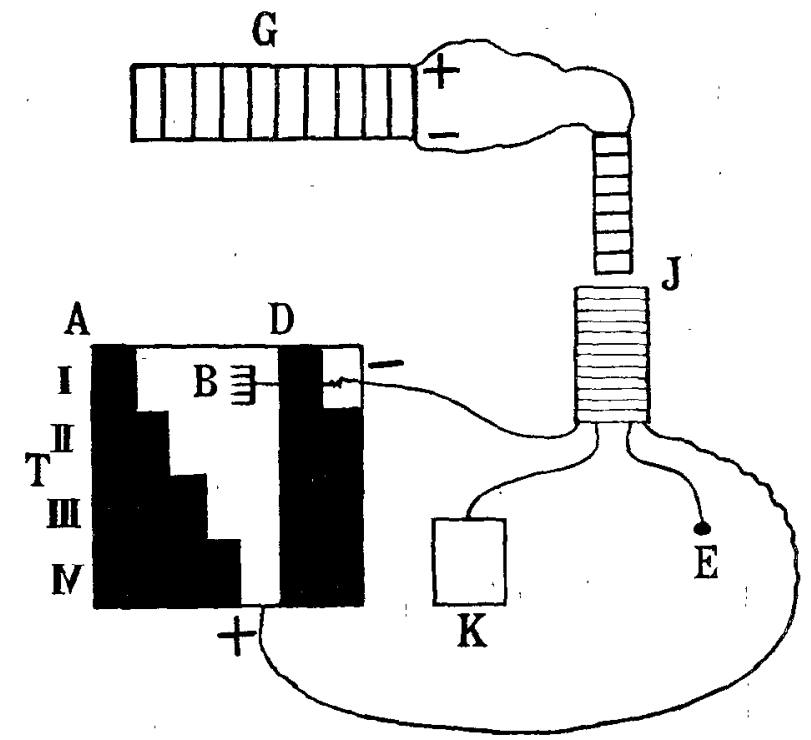

Fig. 5. (Schema der Versuchsanordnung.) $G$ Thermosäule. $J$ Schlitteninduktorium. $K$ Indifferente Elektrode. $E$ Reizelektrorle. $T$ Trommelinterruptor. $B$ Metallbürste.

IV bestrich. So erhielten wir, bei gleicher Rotationsgeschwindigkeit, vier Kombinationen von Reizdauer und Intervall. Durch Veränderung der Umlaufszeit kann man die Kombinationen beliebig vervielfältigen.

\section{Versuchsgruppe A.}

Die Versuchsanordnung ist durch Fig. 3 veranschaulicht. Oberarm vertikal, Unterarm horizontal. Überlastung mit $3 \mathrm{~kg}$. Tatanisierung des Biceps brachii mit Strömen von 7250 E. (Einheiten). 50 Unterbrechungen in 1 Sek. des Primärstromes vom Schlitteninduktorium, das mit einer grossen Gülcher'schen Thermosäule (66 Elemente) armiert war. Die rhythmische Abblendung (Intervall) der tetanisierenden Ströme wird besorgt durch die oben erwähnten 
Trommelstreifen ( $T$ Fig. 3). Die indifferente Elektrode auf der Nabelgegend. Beide Elektroden waren stets gut befeuchtet. Induktionsströme von nahezu maximaler Reizwirkung. Ruhepausen zwischen den Gruppen: je 5 Min. Nur zwischen Gruppe VII und VIII 10 Min. Während jeden Hubes ruht die Schreibtafel. Der Schreibstift schiebt bei jedem Abfalle den Rahmen um je einen Zahn vor.

\begin{tabular}{c|c|c|r|r|r|r}
\hline $\begin{array}{c}\text { Nummer } \\
\text { der } \\
\text { Reizperiode }\end{array}$ & $\begin{array}{c}\text { Reiz- } \\
\text { dauer } \\
\text { Sek. }\end{array}$ & Intervall & $\begin{array}{c}\text { Anzahl } \\
\text { Sek. }\end{array}$ & \multicolumn{2}{|c|}{$\begin{array}{c}\text { Höhe der Hube } \\
\text { (Millimeter) }\end{array}$} & Bemerkungen \\
\hline & & & erster & letzter & \\
II & 0,5 & 2,0 & 6 & 99 & 93 & vgl. Fig. 6 \\
III & 1,0 & 1,5 & 6 & 115 & 100 & \\
IV & 1,5 & 1,0 & 6 & 125 & 94 & \\
V & 2,0 & 0,5 & 1 & 114 & -100 & \\
VI & 1,5 & 2,0 & 7 & 108 & 100 & \\
VII & 1,0 & 1,5 & 8 & 112 & 90 & \\
VIII & 0,5 & 2,0 & 8 & 78 & 69 & \\
IX & 0,5 & 2,0 & 9 & 84 & 81 & \\
X & 1,0 & 1,5 & 9 & 115 & 78 & \\
& 1,5 & 1,0 & 8 & 120 & 81 &
\end{tabular}

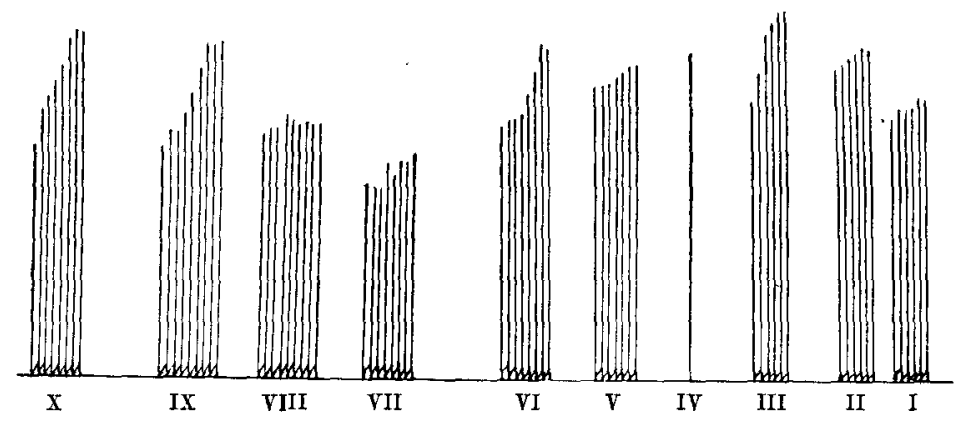

Fig. 6. (Etwa $1 / 8$ der ausgewerteten Hubhöhen.)

Versuchsgruppe B.

Anordnung wie bei Gruppe A; nur werden hier die Reizungen bis zur völligen Ermüdung fortgesetzt.

\begin{tabular}{c|c|c|c|c|c}
\hline $\begin{array}{c}\text { Nummer } \\
\text { der Reiz- } \\
\text { periode }\end{array}$ & $\begin{array}{c}\text { Reizdauer } \\
\text { Sek. }\end{array}$ & $\begin{array}{c}\text { Intervall } \\
\text { Sek. }\end{array}$ & $\begin{array}{c}\text { Anzahl } \\
\text { der Hube }\end{array}$ & $\begin{array}{c}\text { Höhe des } \\
\text { ersten Hubes } \\
\text { mm }\end{array}$ & $\begin{array}{c}\text { Be- } \\
\text { merkungen }\end{array}$ \\
\hline I & 0,5 & 2,0 & 22 & 106 & vgl. Fig. 7 \\
II & 1,0 & 1,5 & 24 & 101 & \\
III & 1,0 & 1,5 & 18 & 108 & \\
IV & 1,5 & 1,0 & 26 & 100 & \\
V & 0,5 & 2,0 & 33 & 78 &
\end{tabular}



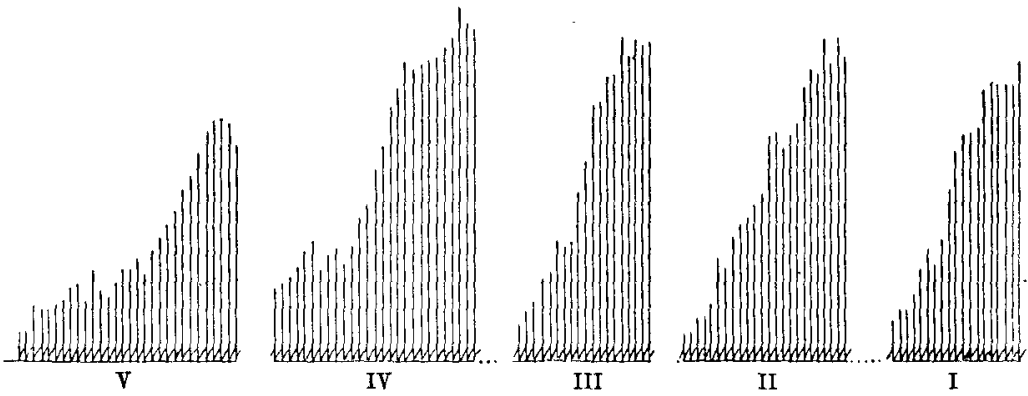

Fig. 7. (Ca. 1/3 der ausgewerteten Hubhöhen.)

Versuchsgruppe C.

Anordnung wie bei Gruppe A und B:

\begin{tabular}{|c|c|c|c|c|c|}
\hline $\begin{array}{l}\text { Nummer } \\
\text { der Reiz- } \\
\text { gruppe }\end{array}$ & $\begin{array}{l}\text { Reizdauer } \\
\text { Sek. }\end{array}$ & $\begin{array}{c}\text { Intervall } \\
\text { Sek. }\end{array}$ & $\begin{array}{c}\text { Anzahl } \\
\text { der Hube }\end{array}$ & $\begin{array}{c}\text { Maximale } \\
\text { Höhe } \\
\text { mm }\end{array}$ & Bemerkungen \\
\hline $\begin{array}{c}\text { I } \\
\text { III } \\
\text { IV } \\
\text { V } \\
\text { VI }\end{array}$ & $\begin{array}{l}0,5 \\
1,0 \\
1,5 \\
1,5 \\
1,0 \\
0,5\end{array}$ & $\begin{array}{l}2,0 \\
1,5 \\
1,0 \\
1,0 \\
1,5 \\
2,0\end{array}$ & $\begin{array}{l}38 \\
36 \\
34 \\
31 \\
32 \\
41\end{array}$ & $\begin{array}{r}94 \\
114 \\
124 \\
107 \\
111 \\
52\end{array}$ & $\begin{array}{l}\text { Die Reizelektrode } \\
\text { war nicht ganz } \\
\text { feucht }\end{array}$ \\
\hline
\end{tabular}

Resultate der Versuchsgruppen A, B und C.

Die günstigste Kombination ist folgende: 1,5 Sek. Reiz, 1 Sek. Intervall. Dieses Verhältnis gilt aber nicht für jedes Gewicht und jede Stromstärke. Wir werden noch darauf zu sprechen kommen.

Taskinen ${ }^{1}$ ) fand bei einem Reizintervall von 2. Sek. den blutdurchströmten Froschmuskel ungefähr gleich schnell ermüdet wie den blutleeren. Zur vollständigen Frholung waren 3-6 Stunden Ruhe erforderlich.

\section{Bestimmung der maximalen Reize.}

Die meisten Experimentatoren auf diesem Gebiete klagen darüber, dass es infolge der grossen Schmerzhaftigkeit unmöglich sei, irgendwie starke Muskelreizungen anzuwenden. Auch mir waren die Versuche anfänglich ein Martyrium, aber auch hier half die Selbst-

1) Beiträge zur Kenntnis der Ermüdung des Muskels. Skandin. Arch. f. Physiol. Bd. 23 S. 1. 
überwindung und Gewöhnung. Anfänglich empfand ich Reize von 3000 E. schon als sehr schmerzhaft, während ich nach 3 Monaten Schläge von $13000 \mathrm{E}$. (die maximale Spannung eines grossen Induktoriums) ertragen lernte.

Versuchsgruppe D.

Anordnung wie bei Versuchsgruppe A. Ruhepausen 1 Min., Reizdauer 1,5 Sek., Intervall 1 Sek.

\begin{tabular}{r|r|r|r|r|r|r|r|l}
\hline $\begin{array}{c}\text { Nr. der } \\
\text { Reiz- } \\
\text { periode }\end{array}$ & $\begin{array}{c}\text { Strom- } \\
\text { ein- } \\
\text { heiten }\end{array}$ & $\begin{array}{c}\text { An- } \\
\text { zahl } \\
\text { der } \\
\text { Hube }\end{array}$ & $\begin{array}{c}\text { Hub- } \\
\text { höhen } \\
\text { mm }\end{array}$ & $\begin{array}{c}\text { Nr. der } \\
\text { Reiz- } \\
\text { periode }\end{array}$ & $\begin{array}{c}\text { Strom- } \\
\text { ein- } \\
\text { heiten }\end{array}$ & $\begin{array}{c}\text { An- } \\
\text { zahl } \\
\text { der } \\
\text { Hube }\end{array}$ & $\begin{array}{c}\text { Hub- } \\
\text { höhen } \\
\text { mm }\end{array}$ & Bemerkungen \\
\hline I & 6000 & 4 & 96 & XIII & 10000 & 1 & 90 & Die Kontraktions- \\
II & 7250 & 4 & 98 & XIV & 9000 & 1 & 84 & werte sinken mit \\
III & 8500 & 4 & 100 & XV & 8000 & 1 & 80 & zunehmender \\
IV & 9000 & 4 & 115 & XVI & 7000 & 1 & 65 & Ermüdung (vgl. \\
V & 10000 & 5 & 118 & XVII & 13000 & 1 & 112 & Fig. 8). \\
VI & 11000 & 5 & 108 & XVII & 12000 & 1 & 85 & \\
VII & 10000 & 5 & 95 & XIX & 11000 & 1 & 83 & \\
VIII & 12000 & 5 & 108 & XX & 10000 & 1 & 80 & \\
IX & 11000 & 5 & 96 & XXI & 9000 & 1 & 78 & \\
X & 19000 & 3 & 100 & XXII & 8000 & 1 & 50 & \\
XI & 12000 & 1 & 108 & XXII & 7000 & 1 & 46 & \\
XII & 11000 & 1 & 94 & XXIV & 6000 & 1 & 0 &
\end{tabular}

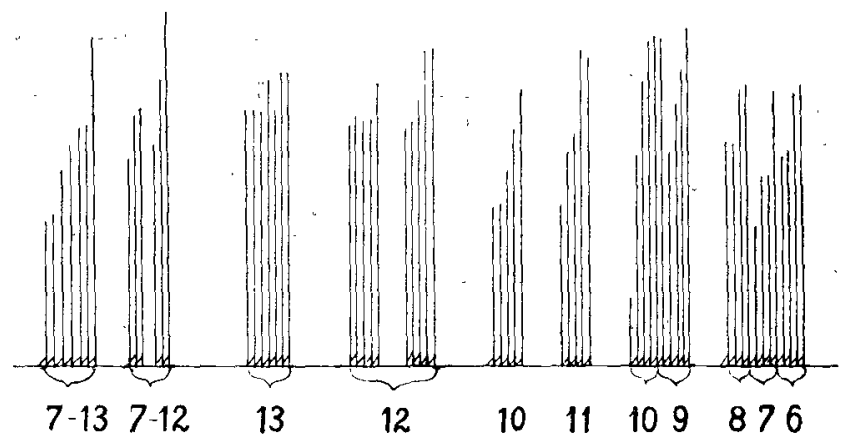

Fig. 8. (Etwa 1/3 der ausgewerteten Hubhöhen.)

Versuchsgruppe E.

Anordnung wie bisher. 50 Unterbrechungen reizten. Gewicht $3 \mathrm{~kg}$, Reizdaner 1,5 Sek., Intervall 1 Sek; Ruhepausen bis Gruppe V: je 2 Min., dann keine mehr. 
Über Ermüdung willkürlich oder elektrisch gereizter Muskeln.

\begin{tabular}{|c|c|c|c|c|c|}
\hline \multirow{2}{*}{$\begin{array}{l}\text { Nummer } \\
\text { der Reiz- } \\
\text { periode }\end{array}$} & \multirow{2}{*}{$\begin{array}{l}\text { Strom- } \\
\text { einheiten }\end{array}$} & \multirow{2}{*}{$\begin{array}{c}\text { Anzabl } \\
\text { der Hube }\end{array}$} & \multicolumn{2}{|c|}{$\begin{array}{l}\text { Höhe der Hube } \\
\text { in Millimetern }\end{array}$} & \multirow[t]{2}{*}{ Bemérkungen } \\
\hline & & & erster & letzter & \\
\hline $\begin{array}{c}\text { I } \\
\text { II } \\
\text { III } \\
\text { IV } \\
\text { V } \\
\text { VI } \\
\text { VII } \\
\text { VIII } \\
\text { IX }\end{array}$ & $\begin{array}{r}8000 \\
8000 \\
8000 \\
8000 \\
8000 \\
10000 \\
11000 \\
12000 \\
13000\end{array}$ & $\begin{array}{r}25 \\
20 \\
22 \\
21 \\
22 \\
4 \\
4 \\
4 \\
1\end{array}$ & $\begin{array}{l}124 \\
117 \\
102 \\
72 \\
63 \\
27 \\
22 \\
17 \\
0\end{array}$ & $\begin{array}{r}9 \\
9 \\
7 \\
6 \\
7 \\
8 \\
10 \\
5 \\
0\end{array}$ & vgl. Fig. 9 \\
\hline
\end{tabular}

Scblussfolgerung: Auch zunehmende Stromstärken vermögen die Ermüdung nicht zu überwinden.

Fig. 9. (Etwa 1/3 der ansgewerteten Hubhöhen.)

Versuchsgruppe F.

Der ganze Arm horizontal gelagert (Bizeps etwas gedehnt). Es werden durch Öffnen des Primärstromes Finzelreize gegeben und zwar in Intervallen von je 0,5 Sek. Schliessungsschläge sind, auch bei höchstmöglicher Intensität (13000 E.), motorisch unwirksam und nur mässig fühlbar. Gewicht $1 \mathrm{~kg}$.

\begin{tabular}{c|c|c|c|c|c}
\hline $\begin{array}{c}\text { Nummer } \\
\text { des Reizes }\end{array}$ & $\begin{array}{c}\text { Strom- } \\
\text { einheiten }\end{array}$ & $\begin{array}{c}\text { Höhe } \\
\text { des Hubes } \\
\text { mm }\end{array}$ & $\begin{array}{c}\text { Nummer } \\
\text { des Reizes }\end{array}$ & $\begin{array}{c}\text { Strom- } \\
\text { einheiten }\end{array}$ & $\begin{array}{c}\text { Höhe } \\
\text { des Hubes } \\
\text { mm }\end{array}$ \\
\hline I & 6000 & 3 & VI & 11000 & 12 \\
II & 7000 & 6 & VII & 9000 & 7 \\
III & 8000 & 9 & VIII & 6000 & 3 \\
IV & 9000 & 10 & IX & 4000 & 2 \\
V & 10000 & 12 & X & 5000 & 2
\end{tabular}

Reizung mit $3000 \mathrm{E}$ ergab keinen Hub mehr. 
Resultate der letzten Versuchsgruppen D, E und F.

1. Der maximale Effekt wird ausgelöst: durch Einzelreize der Intensität $10000 \mathrm{E}$., bleibt aber gering.

2. Auch stärkste Reize werden bald unwirksam.

3. Ströme von $7000-8000 \mathrm{E}$. lösten hohe Leistungen aus, ohne schnell zu ermüden.

4. Intensivere Reize sind schmerzhaft, ohne die Leistung wesentlich zu erhöhen.

5. Gruppe $\mathrm{F}$ zeigte, dass erst $\ddot{\mathrm{O} f \mathrm{f}} \mathrm{n} \mathrm{ng}$ sinduktionsschläge von 4000 E. minimale Zuckungen meines Bizeps auslösten.

6. Die Zuckungen wuchsen mit den Reizen, bis diese $9000 \mathrm{E}$. erreicht hatten; sie werden dann nicht mehr höher, wenn man die Reize verstärkt.

7. Die geleistete Arbeit der Zuckungen ist sehr klein, da nicht mehr als $1 \mathrm{~kg}$ gehoben wurde.

Später werden wir noch über Versuche berichten, bei denen durch eigentümliche Reizbarkeitsänderungen auch schwächere Reize wirksam werden. Einzelne Schliessungsschläge, auch der stärksten uns zu Gebote stehenden Intensität, blieben mechanisch wirkungslos. Gleichwohl haben wir dieselben später bei anderen Versuchen abgeblendet, um ihren etwaigen Einfluss auf die Reizbarkeit auszuschalten.

\section{V. Über die günstigste Reizfrequenz.}

Um diese Frage zu studieren, mussten wir unsere Methodik etwas modifizieren. Zunächst wurde in die Bahn der primären Spirale des Induktorium K ronecker's Interruptor eingeschaltet, der 2- bis $60 \mathrm{mal}$ in der Sekunde den Strom unterbricht und zugleich eine Vorrichtung besitzt, um die Schliessungsschläge abzublenden. Um den Verlauf der Kontraktionen zu registrieren, wurde eine berusste Kymographiontrommel an den Bleistift gebracht. Ein Zeitschreiber markierte 0,1 Sekunde unter die Kurven.

$$
\text { Versuchsgruppe G. }
$$

Der Bizeps des horizontal gelagerten Oberarmes wurde mittels tetanisierender Öffnungsinduktionsströme verschiedener Erequenz gereizt. Die Schliessungsschläge waren durch Nebenschliessung am Unterbrecher abgeblendet. Die Tetanisierung wurde fortgesetzt, bis die Zusammenziehung nicht mehr zunahm (zuweilen auch etwas 
Über Ermüdung willkürlich oder elektrisch gereizter Muskeln.

länger.) Diese Zeit ist im Tabellenkopfe als Summationszeit bezeichnet. Gewicht $1 \mathrm{~kg}$. Grössere Gewichte wurden bei geringer Reizfrequenz vom Biceps nicht merklich gehoben. Ruhepausen zwischen den Reizperioden: 3 Min. Drei Unterbrechungen des Primärstromes ergaben kleine Zuckungen, aber noch keine Summation.

\begin{tabular}{|c|c|c|c|c|c|c|c|}
\hline \multirow{2}{*}{$\begin{array}{l}\text { Nummer } \\
\text { der Reiz- } \\
\text { perioden }\end{array}$} & \multirow{2}{*}{$\begin{array}{c}\text { Strom- } \\
\text { einheiten }\end{array}$} & \multirow{2}{*}{$\begin{array}{c}\text { Frequenz } \\
\text { der } \\
\text { Unterbre- } \\
\text { chungen }\end{array}$} & \multirow{2}{*}{$\begin{array}{c}\text { Reiz } \\
\text { dauer } \\
\text { Sek. }\end{array}$} & \multicolumn{2}{|c|}{$\begin{array}{l}\text { Summationszeit } \\
\text { (Sekunden) }\end{array}$} & \multicolumn{2}{|c|}{$\begin{array}{l}\text { Maximale Höhe } \\
\text { in Millimeter }\end{array}$} \\
\hline & & & & $\begin{array}{c}\text { bis zur } \\
\text { ersten } \\
\text { Erhebung }\end{array}$ & $\begin{array}{c}\text { bis zum } \\
\text { Maxi- } \\
\text { mum }\end{array}$ & $\begin{array}{c}\text { der } \\
\text { ersten } \\
\text { Erhebung }\end{array}$ & $\begin{array}{l}\text { des } \\
\text { Kurven- } \\
\text { gipfels }\end{array}$ \\
\hline I & 7000 & 3 & 10 & - & - & - & - \\
\hline II & 7000 & 4 & 10 & - & 10 & - & 15 \\
\hline III & 7000 & 4 & 10 & - & 9 & - & 14 \\
\hline IV & 7000 & 6 & 7 & - & 4 & - & 16 \\
\hline $\mathrm{V}$ & 7000 & 6 & 6 & - & 3 & - & 16 \\
\hline VI & 7000 & 8 & 13 & 0,3 & 11 & 8 & 30 \\
\hline VII & 7000 & 8 & 12 & 0,3 & 10 & 8 & 33 \\
\hline VIII & 9000 & 8 & 4 & 0,3 & 4 & 18 & 43 \\
\hline IX & 7000 & 13 & 10 & 0,4 & 10 & 15 & 47 \\
\hline $\mathrm{X}$ & 9000 & 13 & 4 & 0,3 & 5 & 25 & 51 \\
\hline $\mathrm{XI}$ & 7000 & 15 & 5 & 0,4 & 5 & 35 & 45 \\
\hline XII & 9000 & 15 & 6 & 0,5 & 5 & 35 & 51 \\
\hline XIII & 7000 & 20 & 3. & 0,5 & 3 & 43 & 52 \\
\hline XIV & 9000 & 20 & 3 & 0,5 & 3 & 47 & 58 \\
\hline $\mathrm{XV}$ & 7000 & 13 & 12 & 0,3 & 5 & 19 & 43 \\
\hline XVI & 7000 & 25 & 3 & 0,8 & 3 & 46 & 54 \\
\hline XVII & 7000 & 50 & 4 & 0,9 & 2,5 & 51 & 59 \\
\hline XVIII & 7000 & 50 & 3 & 0,9 & 2 & 50 & 57 \\
\hline XIX & 7000 & 25 & 4 & 0,8 & 3 & 42 & 53 \\
\hline
\end{tabular}

Versuchsgruppe H.

Anordnung wie bei Versuchsgruppe F. Reiz $7000 \mathrm{E}$.

\begin{tabular}{c|c|c|c|r|c|c|c|c}
\hline \hline $\begin{array}{c}\text { Nr. der } \\
\text { Reiz- } \\
\text { peri- } \\
\text { oden }\end{array}$ & $\begin{array}{c}\text { Fre- } \\
\text { quenz } \\
\text { der } \\
\text { Unter- } \\
\text { brech. }\end{array}$ & $\begin{array}{c}\text { Summa- } \\
\text { tions- } \\
\text { zeit } \\
\text { Sek. }\end{array}$ & $\begin{array}{c}\text { Maxi- } \\
\text { male } \\
\text { Höhe } \\
\text { mm }\end{array}$ & $\begin{array}{c}\text { Nr. der } \\
\text { Reiz- } \\
\text { peri- } \\
\text { oden }\end{array}$ & $\begin{array}{c}\text { Fre- } \\
\text { quenz } \\
\text { der } \\
\text { Unter- } \\
\text { brech }\end{array}$ & $\begin{array}{c}\text { Summa- } \\
\text { tions- } \\
\text { zeit } \\
\text { Sek. }\end{array}$ & $\begin{array}{c}\text { Maxi- } \\
\text { male } \\
\text { Höhe } \\
\text { mm }\end{array}$ & Bemerkungen \\
\hline II & 12 & 10 & 18 & XI & 20 & 4 & 22 & Die Summations- \\
II & 15 & 5 & 20 & XII & $\mathbf{2 5}$ & 3 & 23 & zeit gilt bis zum \\
III & 15 & 6 & 21 & XIII & 30 & 3 & 26 & höchsten Kur- \\
IV & 18 & 4 & 22 & XIV & 40 & 2 & 27 & vengipfel \\
V & 18 & 5 & 20 & XV & 40 & 1,5 & 25 & \\
VII & 18 & 5 & 19 & XVI & 40 & 1,5 & 26 & \\
VII & $\mathbf{1 5}$ & 6 & 18 & XVII & 40 & 1,5 & 25 & \\
VIII & 15 & 6 & 16 & XVIII & 50 & 1 & 25 & \\
IX & 12 & 11 & 13 & XIX & 15 & 6 & 11 & \\
X & 18 & 5 & 21 & & & & &
\end{tabular}


Dass die Werte für die Kurvenhöhen in den Gruppen $\mathrm{H}$ und I niedriger sind als in der Versuchsgruppe $G$, rührt daher, dass die Versuche in $\mathrm{H}$ gemacht wurden, nachdem ich schon einen halben Tag gearbeitet hatte.

Versuchsgruppe I.

Anordnung wie für Versuchsgruppe $H$. Reizung mit versehiedenen Stromstärken.

\begin{tabular}{c|c|c|c|c}
\hline $\begin{array}{c}\text { Nummer } \\
\text { der } \\
\text { Reizperioden }\end{array}$ & $\begin{array}{c}\text { Frequenz } \\
\text { der Unter- } \\
\text { brechungen }\end{array}$ & $\begin{array}{c}\text { Strom- } \\
\text { einheiten }\end{array}$ & $\begin{array}{c}\text { Summations- } \\
\text { zeit } \\
\text { Sek. }\end{array}$ & $\begin{array}{c}\text { Maximale } \\
\text { Höhe } \\
\text { mm }\end{array}$ \\
\hline I & 12 & 7000 & 9 & 23 \\
II & 12 & 10000 & 6 & 26 \\
III & 15 & 7000 & 5 & 29 \\
IV & 15 & 10000 & 2 & 31 \\
V & 15 & 11000 & 0,5 & 33 \\
VI & 15 & 12000 & 0,3 & 33 \\
VII & 30 & 10000 & 1,0 & 32
\end{tabular}

Zur Raumersparnis lasse ich nur einige der 50 Kurven reproduzieren, welche den Effekt verschiedener Reizfrequenzen auf die Kurvenform und Höhe zeigen (siehe Fig. 11 u. 12).

Resultate der Versuchsgruppen G, H und I.

1. Die Summation der Einzelreize beginnt bei der Frequenz 4 pro 1 Sek.

2. Bei grosser Reizfrequenz (von acht Unterbrechungen in 1 Sek. an) bemerkt man einen Anfangsruck (Initialerhebung).

3. Mit steigender Frequenz wächst die Höhe sowohl der Initialerhebung als der ganzen Kurve.

4. Mit steigender Frequenz wächst die Geschwindigkeit der Summation.

5. Die Zeit der Summation bis zum Maximum der Kontraktion ist für jede Frequenz gleich starker Reize konstant.

6. Die Kurvengipfel nehmen mit den Reizstärken zu. Dje Initialerhebung wächst bedeutend rascher als die Maximalhöhe.

7. Die Summationszeit nimmt ab mit steigender Reizstärke.

VI. Eine seltsame Wirkung dauernder Reize.

Ich lag auf einem Operationstische und hatte den rechten Arm (Oberarm und Vorderarm gestreckt) wie gewöhnlich, aber obne dass er mit einem Gewichte verbunden war, in die Rinne gelegt. Einzelne 
Über Ermüdung willkiurlich oder elektrisch gereizter Muskeln.
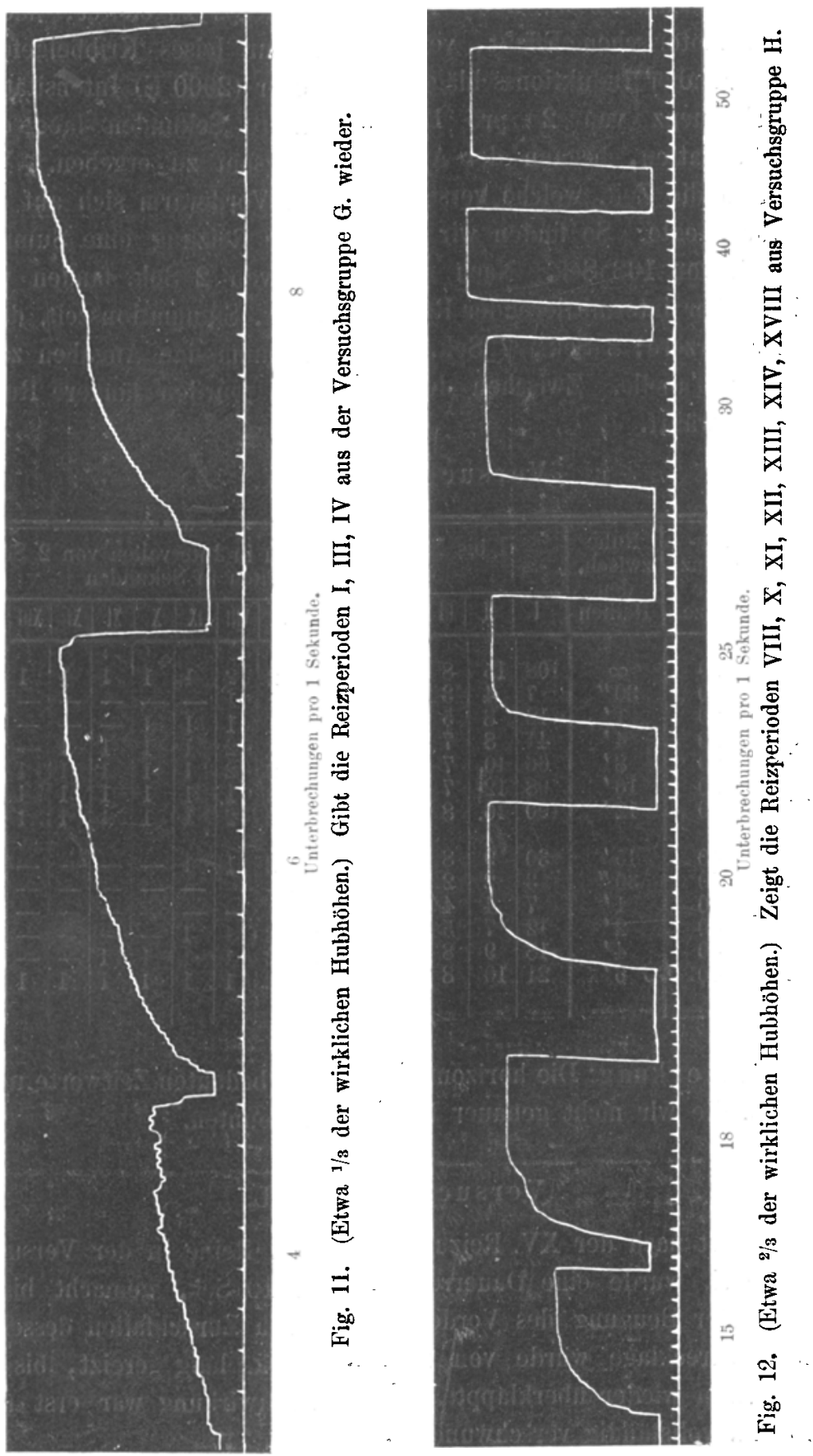

Pflüger's Arehiv für Physiologie. Bd. 116. 
Induktionsschläge von $2000 \mathrm{E}$ dem M. biceps zugeleitet, hatten keinen motorischen Effekt, verursachten nur leises Kribbelgefühl. Nachdem aber Induktionsschläge von gleicher $(2000 \mathrm{E})$ Intensität in der Frequenz von 20 pro 1 Sek. mehrere Sekunden lang eingewirkt hatten, begann der Arm sich langsam zu ergeben. Wir notierten die Zeit, welche verstrich, bis der Vớrderarm sich auf den Oberarm legte. So finden wir für die erste Reizung eine Summationszeit von 108 Sek. Nach einer Pause von 2 Sek. fanden wir, bei gleicher intermittierender Reizung 15 Sek. Summationszeit, dann imımer kürzere: 8 Sek:, 7 Sek. usw. Zahlenmässige Angaben zeigt folgende Tabelle. Zwischen den Reizreihen wurden längere Ruhezeiten gewährt.

Vểrsuchsgruppe K.

\begin{tabular}{|c|c|c|c|c|c|c|c|c|c|c|c|c|c|c|c|c|c|}
\hline \multirow{2}{*}{$\begin{array}{l}\text { Nummer } \\
\text { der } \\
\text { Versuchs- } \\
\text { reihe. }\end{array}$} & \multirow{2}{*}{$\begin{array}{l}\text { Fre- } \\
\text { quenz } \\
\text { der } \\
\text { Reize }\end{array}$} & \multirow{2}{*}{$\begin{array}{l}\text { Ruhe } \\
\text { zwisch. } \\
\text { den } \\
\text { Reihen }\end{array}$} & \multicolumn{15}{|c|}{$\begin{array}{l}\text { I bis XV Reizperioden in Intervallen von } 2 \text { Sek. } \\
\text { Summationszeiten in Sekunden }\end{array}$} \\
\hline & & & $\because 1$ & III & III & 17 & $\bar{V}$ & $\mathrm{Fl}$ & VII & VIII & IX & $\bar{X}$ & $\overline{\mathrm{II}}$ & XII & XIII & XIF & $X V$ \\
\hline I & 20 & & 108 & 15 & & 7 & & 2 & 2 & 2 & 1 & 1 & 1 & 1 & 1 & 1 & \\
\hline II & 20 & $30^{\prime \prime}$ & 7 & 4 & & 3 & & 2 & $\overrightarrow{1}$ & 1 & -1 & - & -1 & - & $=1$ & - & - \\
\hline III & 20 & $1^{\prime}$ & 12 & 8 & & 4 & & 2 & 1 & 1 & 1 & 1 & - & -1 & -1 & - & - \\
\hline IV & 20 & $4^{\prime}$ & 41 & 8 & & & 4 & 2 & 2 & 2 & 1 & 1 & 1 & 1 & -1 & - & - \\
\hline$\pi$ & & $8^{\prime}$ & 66 & 10 & & & 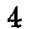 & 3 & 2 & $\overline{2}$ & 1 & 1 & 1 & $\overline{1}$ & 1 & 1 & \\
\hline VI & 20 & $10^{\prime}$ & 98 & 12 & & 5 & 3 & 3 & 2 & 1 & 1 & 1 & 1 & 1 & 1 & 1 & 1 \\
\hline VII & 20 & $12^{\prime}$ & 100 & 16 & & 5 & 3 & 3 & 2 & 2 & 1 & 1 & 1 & 1 & 1 & 1 & 1 \\
\hline & 40 & 15 & 30 & 9 & & 5 & 3. & 2 & 2 & 1 & 1 & - & - & - & - & - & - \\
\hline IX & 40 & $30^{\prime \prime}$ & 3 & 2 & & 1 & & - & - & - & - & - & - & - & - & - & 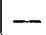 \\
\hline X & 40 & $1^{\prime}$ & 7 & 4 & & 3 & 2: & 1 & 1 & - & - & - & - & -1 & -1 & - & - \\
\hline $\mathrm{X}$ & & $2^{\prime}$ & 12 & 7 & & 3 & a & 2 & 2 & 1 & 1 & - & -1 & - & - & - & 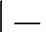 \\
\hline & 44 & $4^{\prime}$ & 18 & 9 & & 5 & 3 & 2 & 2 & 1 & 1 & 1 & 1 & - & -1 & - & - \\
\hline $\mathrm{X}$ & 40 & $6^{\prime}$ & 21 & 10 & & 5 & 3 & 2 & 2 & 1 & 1 & 1 & $\hat{1}$ & 1 & 1 & 一 & - \\
\hline
\end{tabular}

Anmerkung: Die horizontalen Striche bedeuten Zeitwerte unter 1 Sek., die wir nicht genauer bestimmen konnten.

Versuchsgruppe L.

Sofort nach der XV. Reizung der XIII. Reihe in der Versuchsgruppe $\mathrm{K}$, wurde eine Dauerreizung von 10 Sek, gemacht bis zu vollendeter Beugung des Vorderarms. Nach Zurückfallen desselben in die Strecklage wurde von neuem 3 Sek. lang gereizt, bis der Vorderarm wieder überklappte. Diese Nachwirkung war erst nach 15 Min. vollständig verschwunden. 
Resultate der Versuchsgruppen $K$ und L.

1. Intermittierende Induktionsströme von 2000 Einheiten und $1 / 40 "$ bis $1 / 20 "$ Intervall, dem N. Musculocutaneus zugeleitet, lösen während langer Summationszeit ( 30 bis 108 Sek.) eine allmählich zunehmende Bewegung des Vorderarms aus.

2. Die summierten Erregungen wirken während der Ruhepausen bis zu 15 Minuten nach, derart, dass die Summationszeiten kürzer bleiben als nach längerer Ruhe.

3. Die Summationszeit wird kürzer, wenn die Reizfrequenz gesteigert wird.

4. Die Erregbarkeit bleibt kürzere Zeit vermehrt, wenn die Reize frequenter waren ( 40 pro 1 Sek.), als wenn sie seltener folgten, 20 pro 1 Sek.

\section{Versuch M.}

Es war wichtig zu wissen, an welcher Stelle des Nervensystems die Erregbarkeit erhöht wird. $\mathrm{Zu}$ diesem Zwecke mussten Tierexperimente dienen. Einem Kaninchen wurde die Kniesehne des Quadriceps femoris mit einem Schreibhebel verbunden. Die Haut über dem Muskelbauche wurde von Haaren befreit, ebenso eine grössere Fläche auf dem Rücken des Tieres. Die Eintrittsstelle des Astes vom Zentralnerven, der den Rectus femoris versorgt, fixierten wir, ähnlich wie über meinem Biceps brachii mittels zirkulären Heftpflasterstreifens eine kleine Knopfelektrode und befestigten die plattenförmige, indifferente Elektrode auf die freigelegte Rückenhaut des Tieres. Mittels Induktionsströmen von 2 bis $300 \mathrm{E}$ in der Frequenz von 50 pro 1 Sek. folgend, erhielten wir Tetani, deren Summationszeit sich bei jeder folgenden Reizung zu verkleinern schien. Diese Pause zwischen den Reizungen betrug 2 Sek. Leider war es unmöglich, demonstrable Kurven zu erhalten, da das Tier fortwährend willkürliche Zuckungen machte. Wir suchten diese dadurch auszuschalten, dass wir den N, cruralis des gereizten Schenkels durchsehnitten. Wohl verhielt sich das Tier nun ruhig, aber dann war die Steigerung der Erregbarkeit nicht mehr zu sehen.

Dieser freilich nicht beweisende Versuch lässt immerhin annehmen, dass die Tonisierung im Zentralnervensystem (Spinalganglien oder Rückenmarksgrau) geschehe.

Ähnliche Wahrnehmungen machten vor mir schon mehrere Forscher, auf die ich kurz verweisen möchte: 
Minot ${ }^{1}$ ) fand in Boston kurze Tetani frischer Muskeln zuerst an Höhe zunehmend, dann abnehmend. Ermüdete Muskeln erreichen sogleich das Maximum und verlängern sich allmählich.

Hall und. Kronecker ${ }^{2}$ ) beobachteten in Berlin an erregbaren Frühlingsfröschen, dass tetanisierte Muskeln bis 15 Sek. lang nachwirkende Erregung bewahren derart, dass danach ein Induktionsreiz viel höhere Zuckung auslöst als zuvor ein gleicher.

Ähnliches sab $\mathrm{Sewall}{ }^{3}$ ) in Baltimore: "A tetanic stimulus, the excitement due to which has not been allowed to shorten the musele, leaves the muscle in a state of greater irritability toward succeeding stimuli than does a simple stimulus under the same circumstances; moreover the influence of the tetanic stimulation lasts longer than that of the simple one."

\section{VII. Über den Einfluss der Massage auf die Ermüdung.}

Da wir mölicherweise in späteren Versuchen zur Abkürzung der Erholungszeiten dieses Mittel bedürfen, so wollten wir es nicht ungeprüft lassen.

Darüber haben schon Versuche von Kronecker ${ }^{4}$, Tabludowsky ) und Maggiora ${ }^{6}$ ) aufgeklärt. $\mathrm{Hamel}^{7}$ ) hat den Nutzen der Stösse vom arteriellen Blutstrome untersucht und kommt zu dem Schlusse: Der Herzpuls vermittelt eine passive Gymnastik der Arterien und erhält ihre Kohäsion Elastizität und Kontraktibilität.

Wir wünschten den Einfluss der Massage auf die Summationseffekte kennen zu lernen.

1) Experiments of tetanus. Journ. of Anat. and Physiol. vol. 12 p. $297-339$.

2) Die willkürliche Muskelaktion (du Bo is - R eymond's Arch. 1879, Suppl. S. 45.)

3) On the effect of two succeeding stimuli upon muscular contraction. (The Journ. of Physiol. vol. 2 p. 178. 1879-1880.)

4) Kronecker: Ermüdung und Erholung quergestreifter Muskeln. Arbeiten aus der physiol. Anstalt zu Leipzig S. 189. 1871, Ber. der math.-phys. Klasse der Gesellsch. der Wissenschaft zu Leipzig Bd. 23 S. 713.

5) Über die physiologische Bedeutung der Massage. Centralbl. f. d. med. Wissensch. S. 242 u. 243.1883.

6) Action physiologique du massage sur les muscles de l'homme. Mosso Arch. ital. de Biol. t. 13 p. 225. 1891.

7) Die Bedeutung des Pulses für den Blutstrom. Zeitschr. f. Biol. 1889 Bd. 7 S. 495. (Neue Folge.) 
Versuchsgruppe $N$.

Anordnung wie bisher. Der in Ruhe gestreckte Arm, mit $1 \mathrm{~kg}$ belastet, schreibt die Vorderarmbeugungen auf die berusste Trommel. Reizstärke $7000 \mathrm{E}$. Ruhepausen 3 Min., während deren der Arm wiederholt massiert wird. Die Massagen sind in der Tabelle durch Punktlinien markiert.
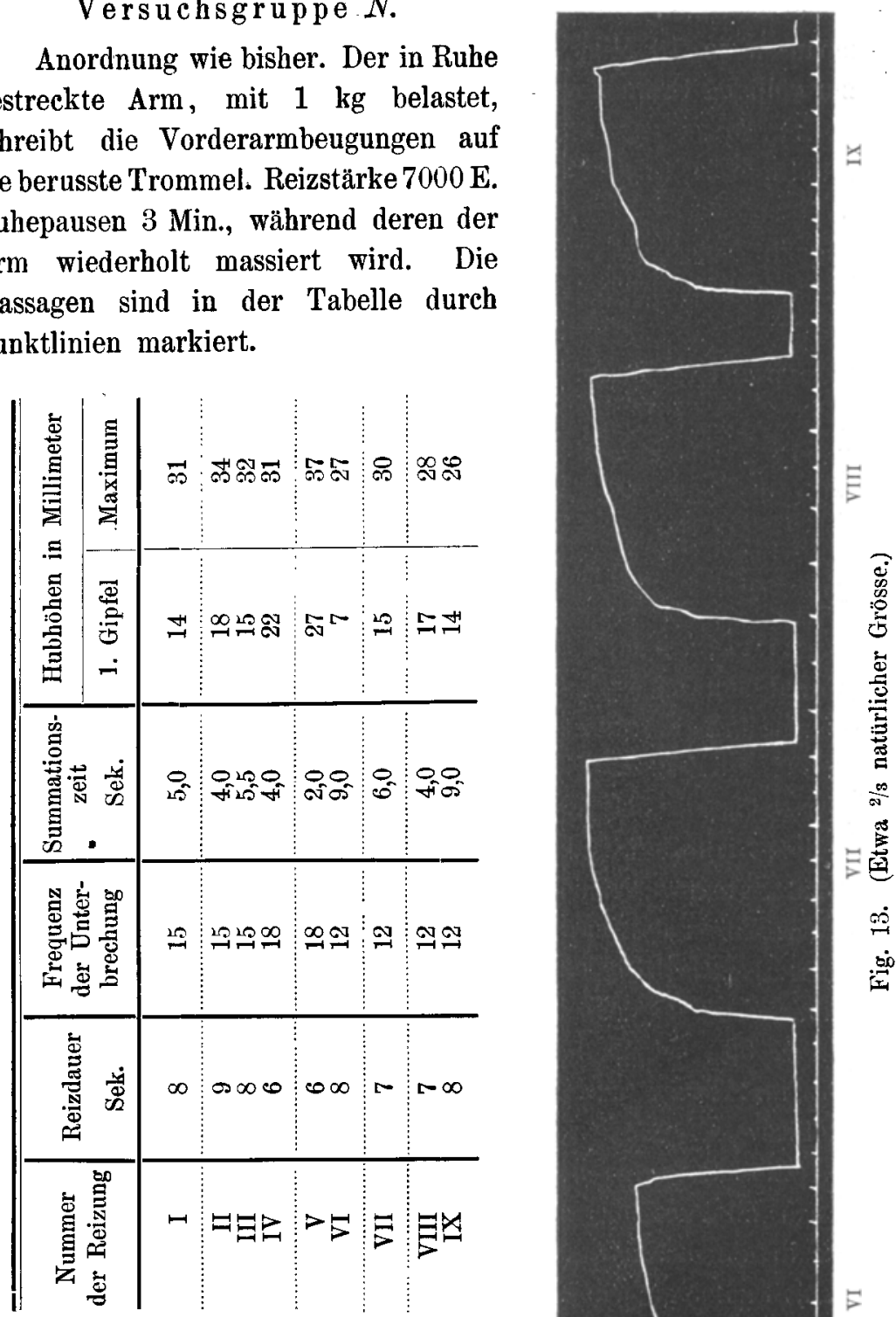

Versuchsgruppe 0 .

Anordnung wie bei Gruppe A mit Schlitteninduktorium; $7000 \mathrm{E}$, 50 Stromunterbrechungen in der Sek. Reizintervalle durch die Isolierstreifen auf der 
Trommel geordnet. Der ganze Arm horizontal gelagert. Gewicht $3 \mathrm{~kg}$. Ruhepausen je 10 Min. Zwischen III und IV Massage. Versuch bis zur völligen Ermüdung fortges etzt (siehe Fig. 14, Tab. auf S. 541.)
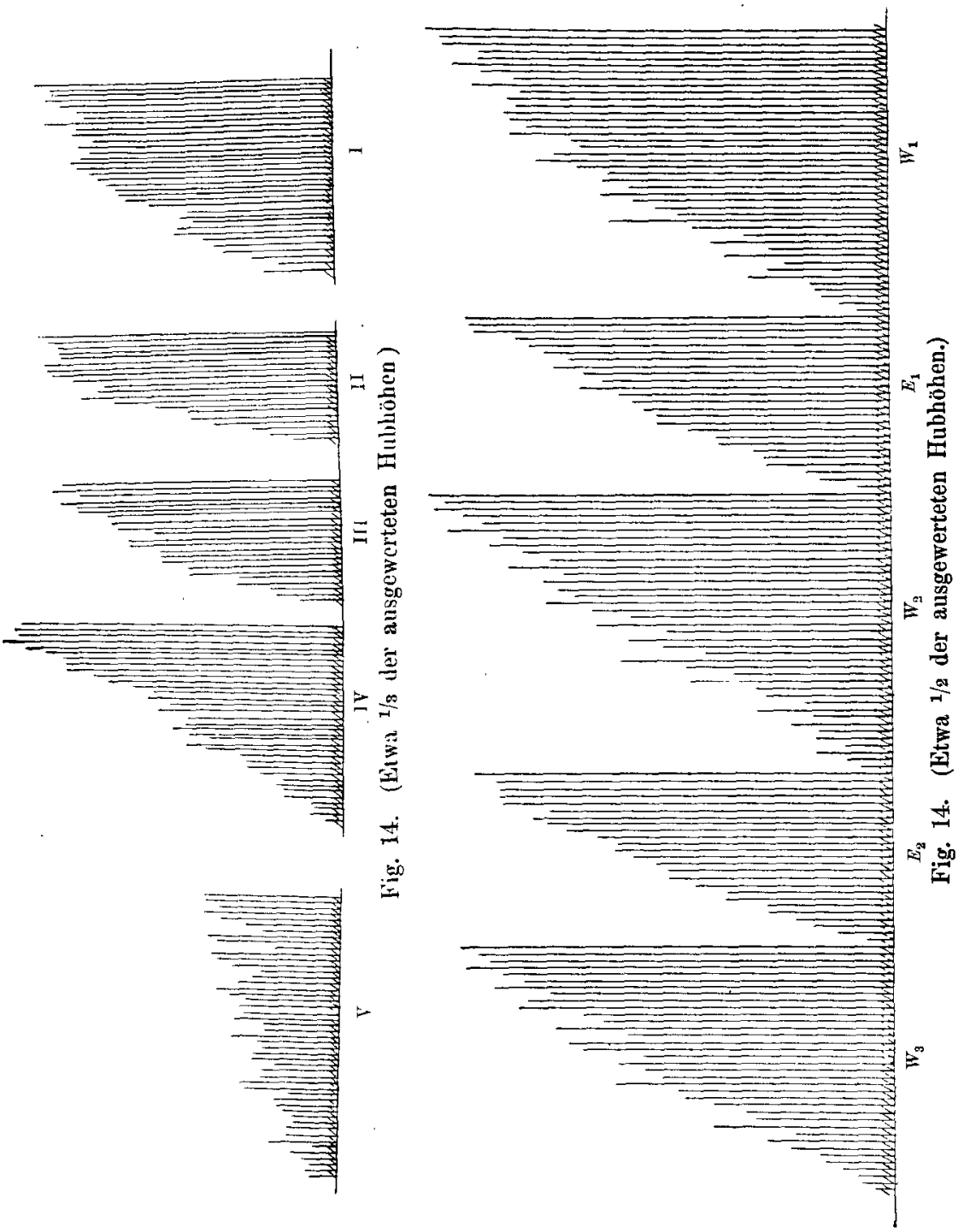

Resultate delr Versuchsgruppen $\mathrm{N}$ unjd 0 .

- 1. Massage beschleunigt die Summationswirkung, ähnlich wie Steigerung der Frequenz (vgl. Fig. 13).

2. Massage vermag nicht nur die Ermüdung aufzuhalten, sondern die Ruhe über die vom unermüdeten Biceps ermöglichten zu bringen. 
Über Ermüdung willkürlich oder elektrisch gereizter Muskeln.

\begin{tabular}{|c|c|c|c|c|c|}
\hline $\begin{array}{c}\text { Nummer } \\
\text { der } \\
\text { Reizung }\end{array}$ & $\begin{array}{c}\text { Reizdauer } \\
\text { Sek. }\end{array}$ & $\begin{array}{c}\text { Intervall } \\
\text { Sek. }\end{array}$ & $\begin{array}{c}\text { Anzahl } \\
\text { der Hube }\end{array}$ & $\begin{array}{l}\text { Höhe des } \\
\text { ersten } \\
\text { Hubes } \\
\text { mm }\end{array}$ & Bemerkungen \\
\hline$\underset{\text { III }}{\text { II }}$ & $\begin{array}{l}1,0 \\
1,5 \\
1,5\end{array}$ & $\begin{array}{l}1,5 \\
1,0 \\
1,0\end{array}$ & $\begin{array}{l}35 \\
22 \\
23\end{array}$ & $\begin{array}{l}122 \\
125 \\
111\end{array}$ & \\
\hline IV & $\begin{array}{l}1,0 \\
0,5\end{array}$ & $\begin{array}{l}1,5 \\
2,0\end{array}$ & $\begin{array}{l}35 \\
49\end{array}$ & $\begin{array}{r}124 \\
56\end{array}$ & $\begin{array}{l}\text { Die Elektroden wa- } \\
\text { ren fast getrocknet }\end{array}$ \\
\hline
\end{tabular}

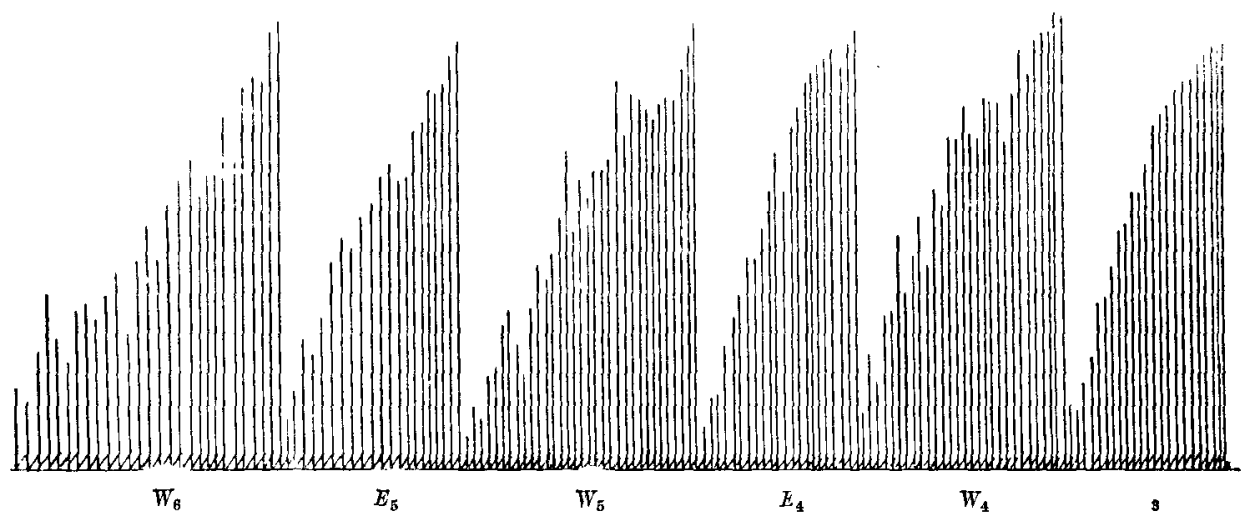

Fig. 14b. (Auf $1 / 2$ der ausgewerteten Hubhöhen.)

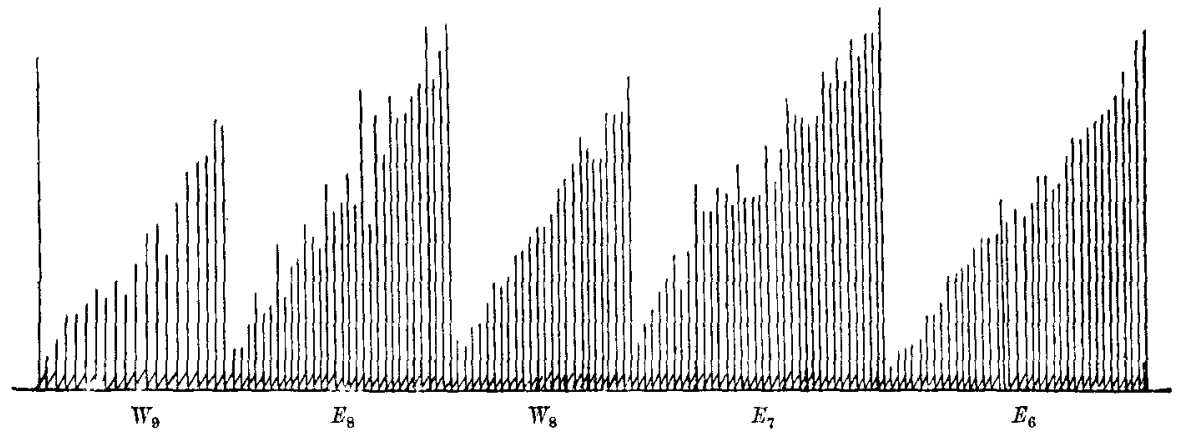

Fig. 14c. (Auf $1 / 2$ der ausgewerteten Hubhöhen.)

VIII. Die Wechselbeziehungen zwisehen willkürlichen und künstlichen Reizen.

Zuerst wurde der M. biceps willkürlich ermüdet, bis er das Gewicht nicht mehr zu heben vermochte, dann erst wurde der Muskel, ohne Zeitverlust, elektrisch gereizt: durch Öffnung der Nebenschliessung der sekundären Spirale des Induktoriums. Die 
542 F. Uhlmann: Über Ermüdung willkürl. oder elektr. gereizter Muskeln.

Reizung wurde so lange fortgesetzt, bis der Biceps völlig ermüdet war: hierauf wurde ohne Pause willkürliche Hebung des Vorderarms versucht.

Versuchsgruppe P.

Vollständige Ermüdungsreihen. Abwechselnd willkürliche und elektrische Reizung. Willkürlich : jede zweite Sekunde ein Hub. (Nach dem Schlage des Metronoms). Elektrisch: 1,5 Sek. Reiz, 1,0 Sek. Intervall: Keine Ruhepausen zwischen den Ermüdungsperioden. Gewicht $2 \mathrm{~kg}$. Strom $7000 \mathrm{E}$. Frequenz 50 Unterbrechungen.

\begin{tabular}{|c|c|c|c|c|c|c|c|c|c|}
\hline \multirow[t]{2}{*}{ Reihe } & \multirow[t]{2}{*}{ Reizart } & \multirow{2}{*}{$\begin{array}{l}\text { Anzahl } \\
\text { der } \\
\text { Hube }\end{array}$} & \multicolumn{2}{|c|}{$\begin{array}{l}\text { Hubhöhe } \\
\text { (Millimeter) }\end{array}$} & \multirow[t]{2}{*}{ Reihe } & \multirow[t]{2}{*}{ Reizart } & \multirow{2}{*}{$\begin{array}{c}\text { Anzahl } \\
\text { der } \\
\text { Hube }\end{array}$} & \multicolumn{2}{|c|}{$\begin{array}{l}\text { Hubböhe } \\
\text { (Millimeter) }\end{array}$} \\
\hline & & & erster & etzter & & & & erster & letzter \\
\hline $\begin{array}{l}W_{1} \\
E_{1} \\
W_{2} \\
E_{2} \\
W_{3} \\
E_{3} \\
W_{4} \\
E_{4}\end{array}$ & $\begin{array}{l}\text { willkürlich } \\
\text { elektrisch } \\
\text { willkürlich } \\
\text { elektrisch } \\
\text { willkürlich } \\
\text { elektrisch } \\
\text { willkürlich } \\
\text { elektrisch }\end{array}$ & $\begin{array}{l}44 \\
26 \\
41 \\
35 \\
27 \\
23 \\
30 \\
30\end{array}$ & $\begin{array}{r}127 \\
102 \\
110 \\
99 \\
103 \\
94 \\
93 \\
93\end{array}$ & $\begin{array}{r}11 \\
9 \\
9 \\
9 \\
8 \\
7 \\
13 \\
13\end{array}$ & $\begin{array}{l}W_{5} \\
E_{5} \\
W_{6} \\
E_{6} \\
W_{7} \\
E_{7} \\
W_{8} \\
E_{8} \\
W_{9}\end{array}$ & $\begin{array}{l}\text { willkürlich } \\
\text { elektrisch } \\
\text { willkürlich } \\
\text { elektrisch } \\
\text { willkürlich } \\
\text { elektrisch } \\
\text { willkürlich } \\
\text { elektrisch } \\
\text { willkürlich }\end{array}$ & $\begin{array}{r}33 \\
20 \\
30 \\
38 \\
36 \\
26 \\
30 \\
19 \\
1\end{array}$ & $\begin{array}{l}93 \\
90 \\
94 \\
77 \\
82 \\
66 \\
78 \\
56 \\
70\end{array}$ & $\begin{array}{r}9 \\
10 \\
11 \\
7 \\
10 \\
9 \\
9 \\
10 \\
-\end{array}$ \\
\hline
\end{tabular}

Resultate der letzten Versuchsreihe.

1. Nach einer Reihe von willkürlichen Huben, bis zur völligen Ermüdung (Leistungsunfähigkeit), vermögen elektrische (intermittierend tetanisierende) Reize (1/50 Sek. Intervall) eine lange Hubreihe anzuregen. Sogleich danach vermochte ich willkürlich fast gleichviel zu leisten wie vor der elektrischen Arbeitsperiode usw.

2. Die Anfangshöhe jeder folgenden Gruppe gleicher Reizart ist niedriger als die frühere.

3. Willkürlich vermochte ich den Arm etwas höher und meist öfter $\mathrm{zu}$ heben als mittels elektrischer Reizung.

4 Die Ermüdungsreihe der Hebungen auf elektrische Reize verläuft regelmässiger als diejenigen bei willkürlichen Hebungen.

5. Während elektrischer Reizung vermochte ich den Vorderarm willkürlich nicht $\mathrm{zu}$ beugen. 